\title{
图神经网络下的生成式室内家具摆放
}

\author{
杨沝 ${ }^{1)}$, 㚞庆楠 $\left.{ }^{2}\right)^{*}$, 王玉洁 1$)$, 段岳圻 ${ }^{2)}$, 陈宝权 ${ }^{3)}$ \\ ${ }^{1)}$ (山东大学计算机科学与技术学院 青岛 266237) \\ 2) (Department of Computer Science, Stanford University Stanford CA94305) \\ ${ }^{3)}$ (北京大学前沿计算研究中心 北京 100871) \\ (fqnchina@gmail.com)
}

\begin{abstract}
摘 要：自动的室内家具摆放在家居设计、动态场景生成等应用中具有显著的意义. 传统算法往往通过显式的空间、 语义和功能性上物体之间的关系来理解场景的内部结构, 并进一步辅助室内场景的生成. 随着大规模室内场景数据 集的出现, 提出将零散的输人家具编码进图结构, 并利用图神经网络中迭代的消息传递隐式地学习场景的分布先验. 为了满足家具摆放的多样性, 提出将图神经网络融合进条件式变分自编码器. 通过一个编码器将输人场景嵌人到一 个符合高斯分布的隐变量, 并通过一个生成器将从隐变量采样的场景先验用于条件式的新场景生成. 在 Fu-floor 数 据集上的实验结果表明，与基准算法相比，该算法在生成结果的评价指标最小匹配距离上表现更优. 该算法对于未 来实现场景补全、基于场景图的室内家具摆放等实际应用也具有显式的意义和价值.
\end{abstract}

关键词：场景生成; 家具摆放; 图神经网络; 条件式变分自编码器

中图法分类号: TP391.41

DOI: 10.3724/SP.J.1089.2021.18457

\section{Graph Neural Network for Generative Furniture Arrangement}

\author{
Yang Miao ${ }^{1)}$, Fan Qingnan ${ }^{2)^{*}}$, Wang Yujie ${ }^{1)}$, Duan Yueqi ${ }^{2)}$, and Chen Baoquan ${ }^{3)}$ \\ 1) (Department of Computer Science and Technology, Shandong University, Qingdao 266237) \\ 2) (Department of Computer Science, Stanford University, Stanford CA94305) \\ 3) (Center on Frontiers of Computing Studies, Peking University, Beijing 100871)
}

\begin{abstract}
Autonomous furniture arrangement plays an important role in many computer vision and graphics applications, such as indoor scene design and dynamic scene generation. The traditional approaches leverage the common spatial, semantical and functional object-object relationships to understand the inner structure of the indoor scene and facilitate the scene generation task. Benefited from the large-scale indoor scene dataset, we propose to embed the unstructured furniture into the graph structure, and leverage the graph neural network to iteratively learn the distribution of scene layout. In order to satisfy the diversity of furniture arrangement, we propose to incorporate the graph neural network into a conditional variational autoencoder. It leverages an encoder to input the scene information into a latent vector that represent the Gaussian distribution, and employ a generator to decode the scene layout from the sampled Gaussian noise for conditional new scene generation. Experimentally, we observe better quality of our algorithm compared to various baselines via the minimum matching distance on the public Fu-floor benchmark. The proposed algorithm is important for many practical applications, including scene completion, interior design based on scene graph and so on.
\end{abstract}

收稿日期：2020-06-30; 修回日期：2020-08-31. 基金项目：国家重点研发计划(2019YFF0302900). 杨沝(1996一), 女, 硕士研究 生, 主要研究方向为计算机图形学; 樊庆楠(1991一), 男, 博士, 论文通讯作者, 主要研究方向为计算机图形学、计算机视觉; 王玉洁 (1994一), 女, 博士研究生, 主要研究方向为计算机图形学; 段岳圻(1992一), 男, 博士, 主要研究方向为计算机视觉、机器学习; 陈宝权(1969-), 男, 博士, 教授, 博士生导师, CCF 会士, 主要研究方向为计算机图形学与可视化. 
Key words: scene synthesis; furniture arrangement; graph neural network; conditional variational autoencoder

家具摆放的目的是将一些给定的输人物体合 理地布置在具有特定大小和功能的房间中. 它在 一系列实际应用中都有重要的价值, 如辅助室内 家装设计、提供搬家的家具重置解决方案以及用于 机器人漫游的动态场景生成等. 这个具有挑战性 的问题通常需要专业的室内场景设计经验, 以构 建一个合理的场景布局. 为了解决这个难题, 传统 算法往往通过探索场景中物体之间的空间、语义或 者功能性的关系来理解房间的整体环境, 并将此 类关系融合进可优化的能量方程或者顺序迭代的 深度学习网络.

然而，场景中的家具摆放具有丰富的多样性， 与设计师的技术和经验等也有复杂的关系, 通过 简单的几何规则计算出来的物体之间的显式关系 仅能代表每个房间的独立摆放样式, 不能反映真 实环境中家具摆放的统计规律. 为了解决这个问 题, 本文提出利用深度学习技术从大规模室内场 景数据集中学习场景分布的先验, 以实现自动化 的家具摆放算法. 该算法将用户提供的一系列家 具嵌人一个有向的图结构, 并利用图神经网络进 行迭代的消息计算和更新. 在消息传递的过程中, 图神经网络从无结构的输人信息中建模家具摆放的 规律, 并将更新后的图节点解码为家具在房间中的 位置和朝向, 以形成合理的房间布局. 图神经网络 的隐式结构信息是从大规模室内场景数据集中学习 得到, 反映了场景布局的统计先验, 相较于物体之 间的显式几何关系更利于本文的家具摆放任务.

为了进一步获得家具摆放的多样性, 本文算 法利用条件式变分自编码器将场景分布的先验拟 合进具有高斯分布特性的隐变量, 并通过对隐变 量的随机采样以获得丰富的家具摆放解决方案. 本文算法通过一个编码器从大规模场景数据集中 学习场景分布的先验, 并基于给定的具有特殊属 性的房间和家具, 通过生成器从高斯分布中解码 出多样的家具摆放结果.

本文提出了不依赖于任何物体之间的显式关 系进行家具摆放和场景生成的算法. 实验表明, 本 文的视觉和数值结果皆大幅超越了基线算法.

\section{1 相关工作}

\section{1 室内场景合成}

早期的室内场景合成工作 ${ }^{[1]}$ 通过利用一些伪
物理和语义的约束来摆放物体. Merrell 等 ${ }^{[2]}$ 提出一 系列的室内场景布置规则来满足一些功能性和视 觉性的场景摆放要求, 并进一步通过用户交互推 荐可能的室内家具摆放方案. $\mathrm{Yu}$ 等 ${ }^{[3]}$ 通过将一些人 类环境改造学和人类工程学上的重要因素融人一 个可优化的能量方程, 实现自动的室内场景生成. Fisher 等 ${ }^{[4]}$ 同时在用户提供的案例和数据集中相似 的场景上训练了一个高斯混合模型以学习摆放物 体. 为了增强一个初始的局部场景, $\mathrm{Fu}$ 等 ${ }^{[5]}$ 和 Fisher 等 ${ }^{[6]}$ 通过实现成对的上下文语义关系来推荐 合理的室内场景布局. 为了进一步约束场景生成 的解空间, 其他相关工作分别基于输人的素描 ${ }^{[7]}$ 、 自然语言 ${ }^{[8-9]}$ 、颜色和深度图像 ${ }^{[10-11]}$ 、人类行为 ${ }^{[12]}$ 构建合理的室内场景.

由于大规模室内场景数据集的出现, 如 SUNCG ${ }^{[13]}$, 一些近期的深度学习算法取代了传统 的模型索引算法, 直接学习生成物体的属性和摆 放位置. Ritchie 等 ${ }^{[14]}$ 和 Wang 等 ${ }^{[15-16]}$ 都是通过训练 一个卷积神经网络来编码输人的室内场景俯视图, 并顺序地合成对应的室内场景. $\mathrm{Li}$ 等 ${ }^{[17]}$ 将一个场 景表示为一个分层的树结构, 并学习迭代地解码 场景布置. Zhang 等 ${ }^{[18]}$ 用一个矩阵参数化一个三维 场景, 并学习了一个多层感知机来自动编码场景 的信息. Zhou 等 ${ }^{[19]}$ 通过对查询位置进行物体类别 的预测来增强一个部分初始化的场景. 给定空间 域上物体之间的连接关系, Luo 等 ${ }^{[20]}$ 通过将可微渲 染器融合进一个神经网络以学习将输人的每个物 体合理地摆放在场景中. 与以上的相关工作不同, 本文的目标是在一个给定大小和功能的房间中, 通过学习到的场景先验将一系列家具布置其中. 为了实现这个目标, 本文算法将输人的物体编码 进图结构, 并利用图神经网络学习构建合理的室 内场景布局.

\section{2 场景图的表示方式}

场景图通过一个带有物体属性和物体间连接 关系的结构图代表一个场景. 随着带有场景图标 注的大规模图像数据集 Visual Genome ${ }^{[21]}$ 的出现, 一系列的工作都通过预测场景图实现各种各样的

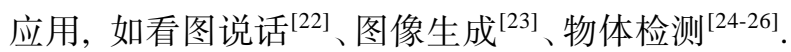
一些与本文更相关的研究工作由于缺少带有标注 的物体之间的连接关系, 便通过一些简单的启发 式策略来生成空间域或者功能性上物体之间的连 接关系, 并以此构建场景图. $\mathrm{Li}$ 等 ${ }^{[17]}$ 学习一个树状 
场景图, 其中, 每种功能性关系参数化为一种具体 的自编码器. Zhou 等 ${ }^{[19]}$ 继承了类似的连接关系并 将其编码进一个基于消息传递的神经网络中以构 建一个场景图. Wang 等 ${ }^{[15]}$ 利用空间位置关系学习 逐渐填补一个空场景图. 与以上工作不同, 本文并 没有利用显式的物体连接关系构建场景图, 而是 通过图神经网络从零散的输人家具中学习结构化 的场景分布先验.

\section{3 生成式模型}

近年来，生成式深度学习模型广泛用于将大 规模的训练数据映射为低维度的隐变量, 并据此 生成符合训练样本规律的数据分布. 目前常用的 基于隐变量的深度学习算法包含生成对抗网络 ${ }^{[27]}$ 和变分自编码器 ${ }^{[28]}$, 其中, 前者通过对抗式分类 器将看似随机的数据分布映射到隐变量中, 而后 者通过一个编码器将一个高斯分布逼近训练数据 样本. 本文采用变分自编码器的一个变种——条 件式变分自编码器 ${ }^{[29-30]}$ 对基于输人房间和家具属 性的房间布局分布进行建模. 与过去的算法不同, 本文提出将图神经网络融合进条件式变分自编码 器, 以满足对场景生成的多样性和质量的要求.

\section{2 本文算法}

\section{1 问题描述和算法概述}

一个房间表示为代表房间的属性物体(如门、 窗和墙壁)的集合, 其中, $N_{\mathrm{r}}$ 为属性物体的数目, $r_{i}$ 表示物体的包围盒在房间的二维平面上的位置、 朝向、长宽和语义信息. 输人的家具集合表示为
$F=\left\{\boldsymbol{f}_{i}\right\}_{i=1}^{N_{\mathrm{f}}}$, 其中, $N_{\mathrm{f}}$ 是家具的数目, $\boldsymbol{f}_{i}$ 表示家具 的包围盒的大小、语义信息和实例标签. 实例标签 用于分区大小和语义标签相同的家具，如成对的 床头柜. 该问题的目标是输出每个家具在特定房 间中的空间位置和朝向 $\boldsymbol{q}_{i}$ 以生成合理的房间布局 $Q=\cup \boldsymbol{q}_{i}$. 因此, 自动的室内家具摆放可以表示为 一个条件式生成式问题, 即在具有特定属性的房 间 $R$ 中，给定输人的家具集合 $F$ ，预测房间布局 $Q$ 的条件式分布.

为了解决这个问题，本文提出一种图神经网 络, 将零散且无结构的输人家具集合编码进一个 有向图, 并通过迭代式的消息传递来建模家具的 摆放规律. 家具摆放本质上是一个多模态问题, 为 了满足该问题输出的多样性, 本文提出利用条件 式变分自编码器学习场景分布的先验. 本文将图 神经网络和变分自编码器融合进一个整体的算法 框架以实现自动化的家具摆放. 算法流程如图 1 所示.

\section{2 图神经网络}

本文将时序 $t$ 上的有向图表示为 $\mathcal{G}^{(t)}=\left(\mathcal{V}^{(t)}\right.$, $\left.\mathcal{E}^{(t)}\right)$. 其中, $\mathcal{V}^{(t)}=\left\{\boldsymbol{h}_{i}^{(t)}\right\}_{i=1}^{N}$ 为图结构中的点集, 为 房间属性物体和家具的隐变量的集合; $h_{i}^{(t)}$ 代表图 中点 $i$ 的隐变量; $\mathcal{E}^{(t)}=\left\{\boldsymbol{m}_{i j}^{(t)}\right\}$ 为图结构中的边集, $\boldsymbol{m}_{i j}^{(t)}$ 代表由点 $i$ 传向点 $j$ 的有向消息. 因此, $N=$ $N_{\mathrm{r}}+N_{\mathrm{f}}$. 由于房间属性物体是固定的, 该问题只 对家具进行预测，因此边集 $\mathcal{E}^{(t)}$ 只包含两两家具之 间的双向关系和房间属性物体对家具的单向关系.

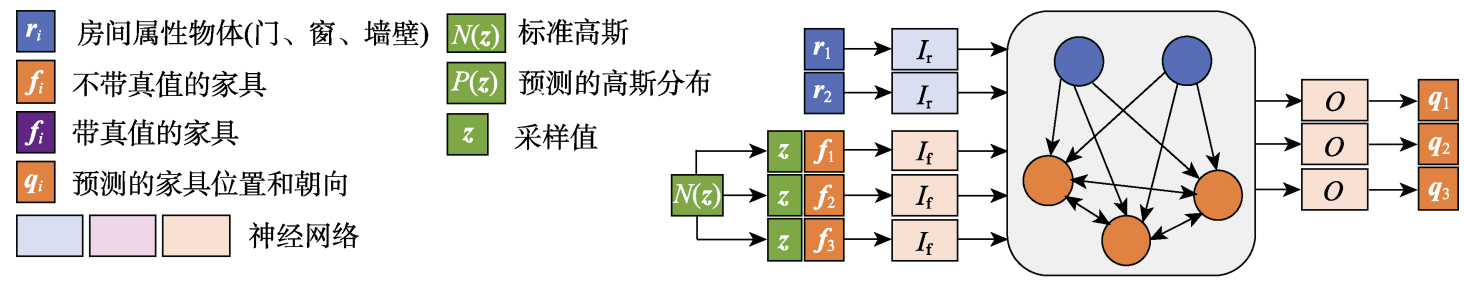

a. 测试阶段

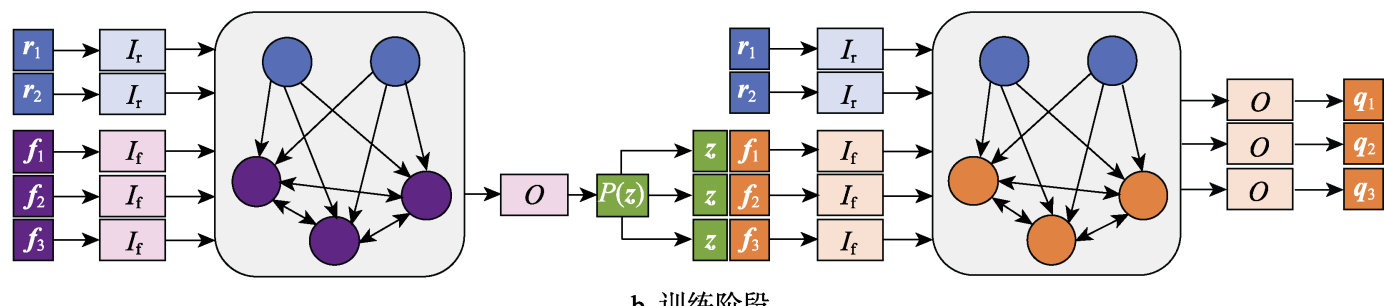

图 1 本文算法流程 
本文将消息传递机制融合进图神经网络. 该 算法包含 3 个阶段：读人、消息传递和读出. 读人 阶段将输人物体的原始信息编码为图神经网络中 的初始隐变量 $\boldsymbol{h}_{i}^{(0)} \in \mathbb{R}^{128}$. 由于房间属性物体和家 具的输人元素不同, 读人函数分别用 2 个神经网络 对其进行编码. 对于房间属性物体而言, 即

对于家具而言，即

$$
\boldsymbol{h}_{i}^{(0)}=I_{\mathrm{r}}\left(\boldsymbol{r}_{i}\right)
$$

$$
\boldsymbol{h}_{i}^{(0)}=I_{\mathrm{f}}\left(\boldsymbol{f}_{\mathrm{i}}\right)
$$

输人物体被表示为二维平面上的包围盒, 其 位置表示为包围盒中心点的 $x y$ 轴坐标, 朝向被量 化为上下左右 4 个方向的独热编码, 大小表示为长 度和宽度, 语义信息表示为长度为 13 的独热编码, 代表 13 个常用的物体类别. 由于房间的属性物体 包含位置、朝向、大小和语义, 因此实现中 $\boldsymbol{r}_{i} \in \mathbb{R}^{21}$; 而家具仅包含大小、语义和实例标签, 因此 $\boldsymbol{f}_{i} \in \mathbb{R}^{16}$, 其位置和朝向属性由算法预测得到.

消息传递分为消息计算 $M$ 和消息更新 $U$. 在 时序 $t+1$, 从点 $j$ 传向点 $i$ 的消息 $\boldsymbol{m}_{j i}^{(t+1)} \in \mathbb{R}^{128}$ 通过 函数 $M$ 计算, 即

$$
\boldsymbol{m}_{j i}^{(t+1)}=M\left(\boldsymbol{h}_{j}^{(t)}, \boldsymbol{h}_{i}^{(t)}\right)
$$

由于房间属性物体在场景中不发生改变，因 此只有家具对应的隐含向量 $\boldsymbol{h}_{i}^{(t+1)}$ 被更新, 即

$$
\boldsymbol{h}_{i}^{(t+1)}=U\left(\boldsymbol{h}_{i}^{(t)}, \sum_{j \in \mathcal{N}(i)} \boldsymbol{m}_{j i}^{(t+1)}\right)
$$

其中， $\mathcal{N}(i)$ 代表所有到达点 $i$ 的邻域节点. 消息 更新函数利用上一轮迭代的隐变量和本轮迭代的 消息总和共同更新本轮的隐变量.

经过 $T$ 轮迭代, 更新后的隐变量被读出函数 $O$ 解码为对应的家具位置和朝向 $\boldsymbol{q}_{i} \in \mathbb{R}^{6}$, 即

$$
\boldsymbol{q}_{i}=O\left(\boldsymbol{h}_{i}^{(T)}, \boldsymbol{h}_{i}^{(0)}\right)
$$

为保持原始的输人信息，避免家具的特征在消 息传递中退化, 读出函数同时输人初始和终止迭代 的隐变量. 上述读人、消息更新和读出函数皆参数 化为 2 层感知机, 其中间特征向量宽度为 256 .

\section{3 生成式家具摆放}

如何将一系列指定的家具合理地摆放在特定 的房间中是一个生成式问题. 该问题的解决方案具 有多样性, 需要学习一个一对多的映射. 为了实现 这个目标, 本文提出利用条件式变分自编码器逼近 家具摆放的数据分布. 在测试阶段，本文算法从一 个常见的已知数据分布(标准高斯分布) $N(z) \in \mathbb{R}^{20 \times 2}$
采样隐变量 $\mathbf{Z}$, 并基于给定的房间属性物体 $R$ 和 家具 $F$, 利用一个生成器 $G$ 预测房间布局. 具体 过程可表示为

$$
Q=G(\mathbf{z}, F, R), \mathbf{z} \sim N(\mathbf{z})
$$

其中, 隐变量 $\mathbf{z}$ 隐含了场景的分布先验; $N(\mathbf{z})$ 代 表 20 个独立的标准高斯的均值和方差; 生成器 $G$ 由第 2.2 节所述的图神经网络构成. 为了实现这个 目的, 将式(2)修改为

$$
\boldsymbol{h}_{i}^{(0)}=I_{\mathrm{f}}\left(\mathbf{z}, \boldsymbol{f}_{i}\right)
$$

其中, 编码家具信息的读人函数 $I_{\mathrm{f}}$ 将家具信息和 采样的隐变量级联在一起作为输人.

为了将场景分布的先验信息嵌人高斯分布, 在训练阶段, 本文算法通过一个编码器 $E$ 将输人 的完整场景信息编码到一个具有高斯分布的潜在 高维空间 $P(\mathbf{z})$ 中, 并将从该空间采样的隐变量输 人生成器 $G$; 而在测试阶段, 从已知的标准高斯 分布 $N(\mathbf{z})$ 采样隐变量. 将其训练阶段具体实现为

$$
\begin{gathered}
P(\mathbf{z})=E\left(F, R, Q^{*}\right) \\
Q=G(\mathbf{z}, F, R), \mathbf{z} \sim P(\mathbf{z})
\end{gathered}
$$

其中, *代表真值信息, 因此 $Q *$ 代表家具的位置和 朝向的真值. 编码器 $E$ 采用第 2.2 节所述的图神经 网络实现. 将编码器的读入函数修改为

$$
\boldsymbol{h}_{i}^{(0)}=I_{\mathrm{f}}\left(\boldsymbol{q}_{i}^{*}, \boldsymbol{f}_{\mathrm{i}}\right)
$$

并将读出函数修改为

$$
P(\mathbf{z})=O\left(\boldsymbol{h}_{0}^{(T)}, \boldsymbol{h}_{1}^{(T)}, \cdots, \boldsymbol{h}_{N}^{(T)}, \boldsymbol{h}_{0}^{(0)}, \boldsymbol{h}_{1}^{(0)}, \cdots, \boldsymbol{h}_{N}^{(0)}\right)
$$

其中, 所有家具的初始和终止隐藏向量级联后经 过一个最大池化层得到一个固定长度的向量, 并 进一步通过一个 3 层感知机输出一个全局的多元 高斯分布 $P(\mathbf{z}) \in \mathbb{R}^{20 \times 2}$. 感知机的中间特征向量的 宽度均为 256 .

\section{4 目标函数}

本文算法的目标函数包含对编码器和生成器 输出的监督. 为了将场景分布的先验拟合进高斯 分布, 本文采用 KL 散度(Kullback-Leibler divergence)目标函数, 表示为

$$
\mathcal{L}_{\mathrm{KL}}=D_{\mathrm{KL}}(P(\mathbf{z}) \| N(\mathbf{z}))=-\int P(\mathbf{z}) \ln \frac{P(\mathbf{z})}{N(\mathbf{z})} \mathrm{d} \mathbf{z}
$$

其中, 编码器输出的潜在高维分布逼近于标准高斯 分布. 为了保持生成结果的质量, 目标函数同时包 含对生成器输出的家具位置 $\boldsymbol{q} \boldsymbol{p}_{i} \in \mathbb{R}^{2}$ 和朝向 $\boldsymbol{q} \boldsymbol{r}_{i} \in \mathbb{R}^{4}$ 的监督. 对家具位置施加 $L_{1}$ 损失函数, 表示为

$$
\mathcal{L}_{\boldsymbol{q} \boldsymbol{p}}=\frac{1}{N_{\mathrm{f}}} \sum_{i=1}^{N_{\mathrm{f}}}\left\|\boldsymbol{q} \boldsymbol{p}_{i}-\boldsymbol{q} \boldsymbol{p}_{i}^{*}\right\|_{1}
$$


家具的朝向约束为上下左右 4 个方向，因此本 文算法对其施加交叉熵损失函数, 即

$$
\mathcal{L}_{\boldsymbol{q}}=\frac{1}{N_{\mathrm{f}}} \sum_{i=1}^{N_{\mathrm{f}}} \boldsymbol{q} \boldsymbol{r}_{i}^{*} \ln \boldsymbol{q} \boldsymbol{r}_{i}
$$

由于家具的摆放位置存在一定程度的歧义性, 本文同时对房间中两两家具之间的相对位置关系 施加了监督, 即

$$
\mathcal{L}_{\text {rel }}=\frac{1}{N_{\mathrm{f}} \cdot N_{\mathrm{f}}} \sum_{i=1}^{N_{\mathrm{f}}} \sum_{j=1}^{N_{\mathrm{f}}}\left\|\left(\boldsymbol{q} \boldsymbol{p}_{i}-\boldsymbol{q} \boldsymbol{p}_{j}\right)-\left(\boldsymbol{q} \boldsymbol{p}_{i}^{*}-\boldsymbol{q} \boldsymbol{p}_{j}^{*}\right)\right\|_{1}
$$

家具摆放的朝向往往由其空间位置决定，因 此 $\mathcal{L}_{\text {rel }}$ 在解决空间位置歧义性的同时也解决了其 摆放朝向的歧义性. 本文曾尝试添加相对朝向关 系和最小化 4 个朝向的监督，但是并未见到明显的 效果提升. 总的目标函数表示为各个损失函数的 加权平均, 即

$$
\mathcal{L}=\mathcal{L}_{\mathrm{KL}}+\lambda_{q p} \mathcal{L}_{q p}+\lambda_{q r} \mathcal{L}_{q r}+\lambda_{\text {rel }} \mathcal{L}_{\text {rel }}
$$

其中, $\lambda_{q p}, \lambda_{q r}$ 和 $\lambda_{\text {rel }}$ 均为预定义的权重标量.

\section{3 实验结果}

\section{1 实现细节}

本文采用 $\mathrm{Fu}$ 等 ${ }^{[5]}$ 采集的室内场景数据集 $\mathrm{Fu}-$
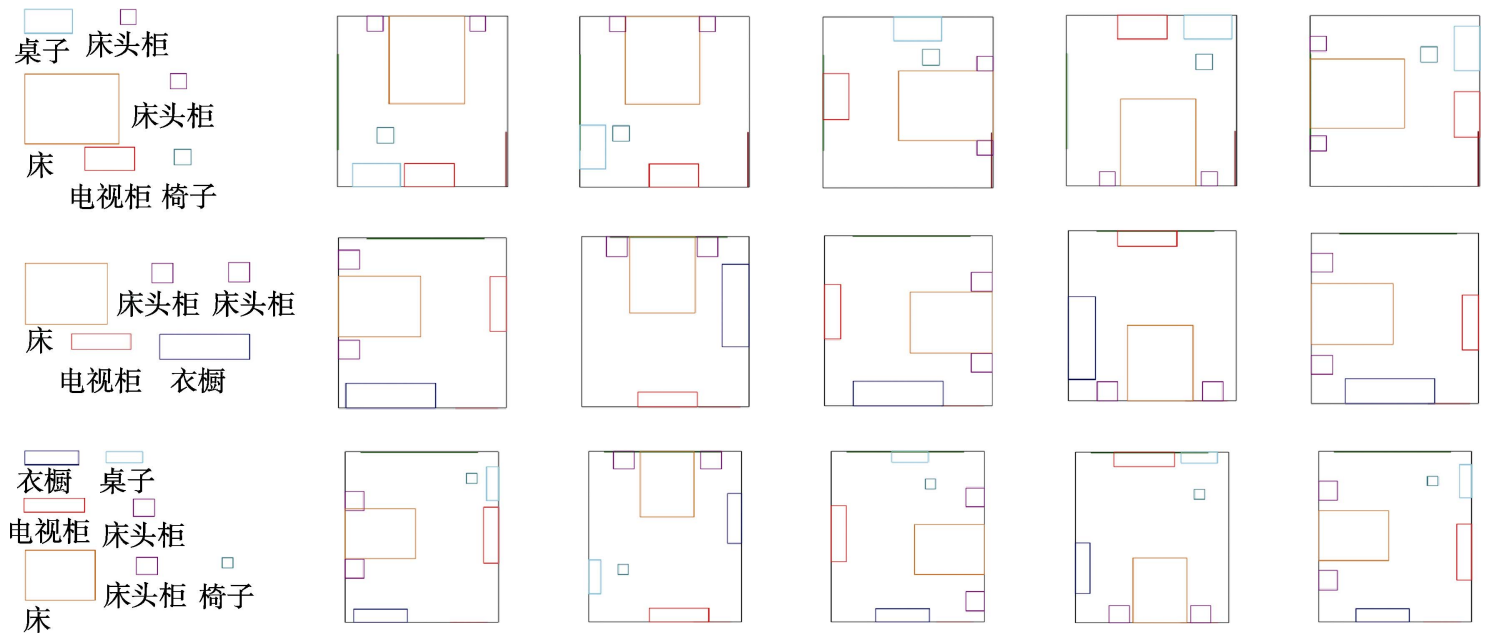

\section{家具(如床头柜)严格对称摆放}

\section{2 家具摆放的生成结果}

为了验证本文算法对场景分布多样性的学习 能力和指定输人家具的摆放能力, 将随机采样的 标准高斯噪声输人生成器, 得到一组家具摆放的 结果, 如图 2 所示. 其中, 同一列不同输人家具的 结果皆由相同的高斯噪声解码生成. 房间包围盒 的大小代表了房间的长宽信息，由输人的房间属 性物体中的墙长度决定.
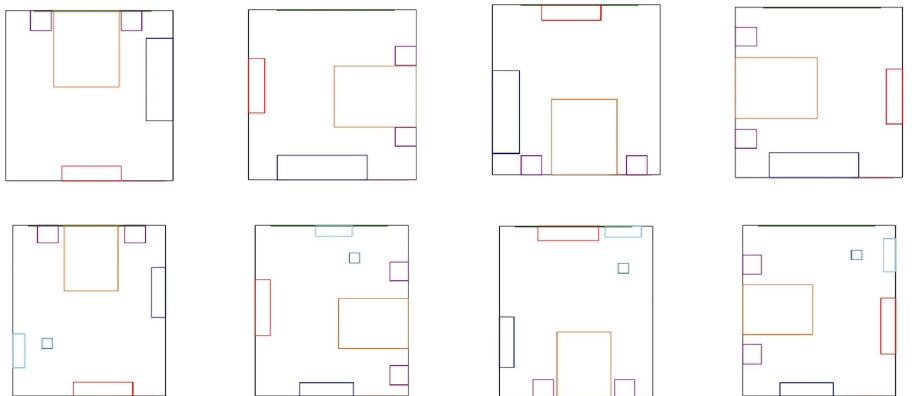

a. 输人家具

b. 家具摆放真值

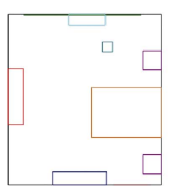

d. 生成结果 2

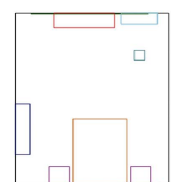

e. 生成结果 3

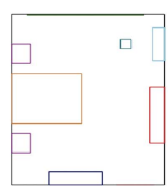

f. 生成结果 4

图 2 家具摆放生成结果

可以看出, 即使输人家具的种类和数目不同, 从相同高斯噪声生成的场景也具有很强的相似性. 这验证了本文算法中条件式变分自编码器学习得 到的隐空间编码了室内场景的分布样式. 另外, 不 同高斯噪声对应的家具摆放各有不同，但也一定
程度覆盖了家具摆放的真值, 这说明本文算法学 习的隐空间兼顾了场景分布的质量和多样性.

\section{3 家具摆放的插值结果}

为了进一步探索隐空间所学到的内容, 本文首 先通过采样 2 组高斯噪声生成合理的家具摆放结果, 
并对 2 组噪声进行线性插值得到中间态的室内场景, 如图 3 所示. 可以看出, 直接插值的结果不具有非

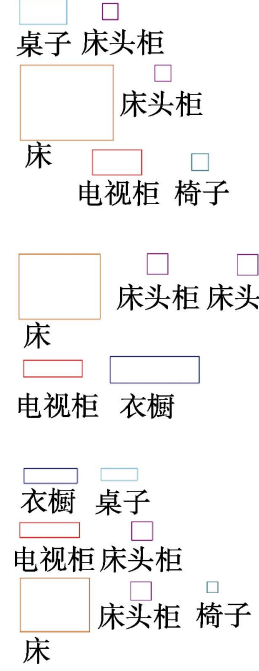

a. 输人家具
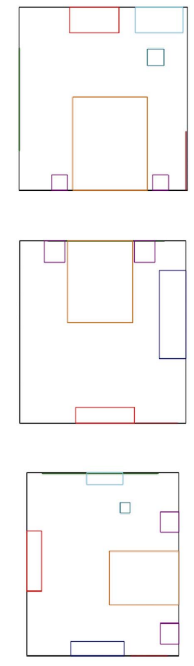

b. 生成结果 1
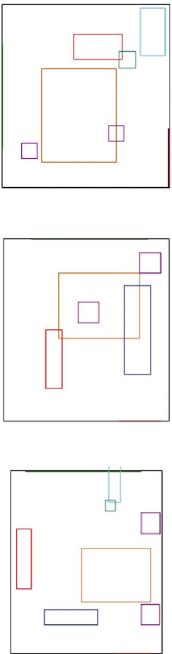

c. 插值结果 1
常合理的室内场景摆放规律，但每个家具的位置和 朝向从生成结果 1 向生成结果 2 渐进地变化.
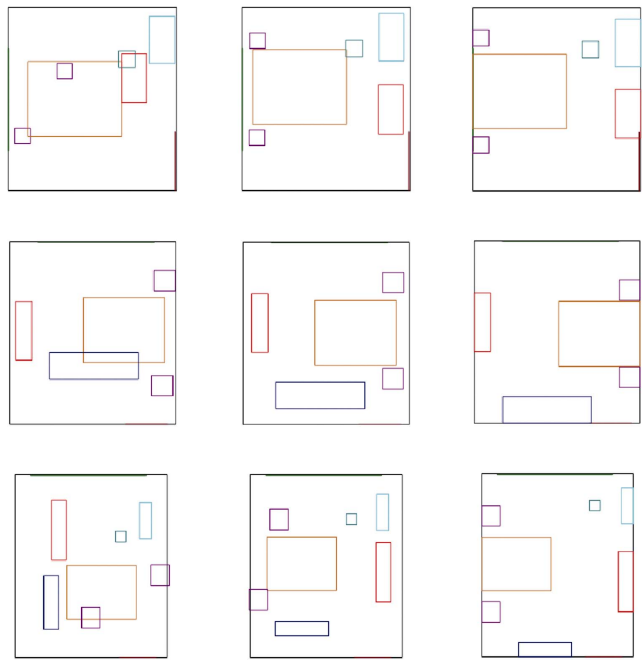

d. 插值结果 2

f. 生成结果 2

图 3 家具摆放的插值结果

\section{4 消融实验}

为了验证本文算法的有效性, 提出 2 种分别针 对网络结构和损失函数的变种算法作为基线算法. 其中，前者将本文的图神经网络替换为简单的多 层感知机. 由于输人的家具数目随着场景的不同 往往在变化, 而感知机的输人大小是固定的, 本文 根据数据集中最大的输人家具数目设置多层感知 机的输人大小, 对不足最大家具数目的场景补零; 后者去掉损失函数中对相对位置关系的监督 $\mathcal{L}_{\text {rel }}$, 以形成一个最简单的损失函数版本.

为了进行有效的数值比较, 使用最小匹配距离 作为测量误差的标准. 对于测试集中的每一个样例, 本文通过采样高斯噪声生成 100 组不同的家具摆放 结果, 并计算其与真值的最小位置和朝向距离 $\left(\mathcal{L}_{q p}+\mathcal{L}_{q r}\right)$ 作为最小匹配距离. 该标准衡量了算法对 于家具摆放真值的匹配程度. 如表 1 所示, 本文算法 取得了最好的数值结果, 效果远远好于其他的基线 算法. 对应的视觉结果如图 4 所示, 可以看出, 本文 算法给出了最好的家具摆放方案，而其他基线算法 的结果都在一定程度上存在家具互相交叉或房间布 局不合理的现象, 进一步显示了本文算法的优越性.

表 1 消融实验的数值结果

\begin{tabular}{cc}
\hline \multicolumn{1}{c}{ 算法 } & 最小匹配距离 \\
\hline 去掉图神经网络 & 0.26 \\
去掉相对损失函数 & 0.29 \\
本文 & $\mathbf{0 . 2 0}$ \\
\hline
\end{tabular}

注. 粗体表示最小匹配距离最优值.

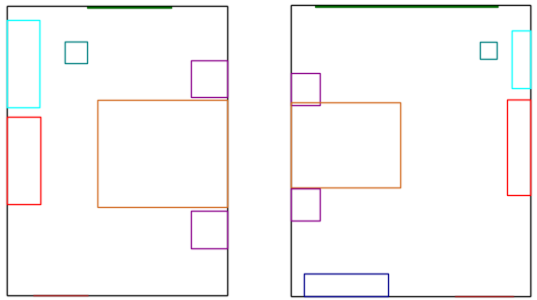

a. 家具摆放真值

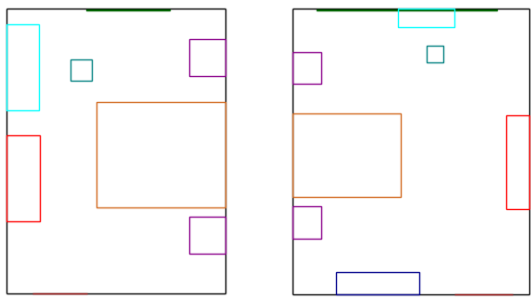

b. 本文

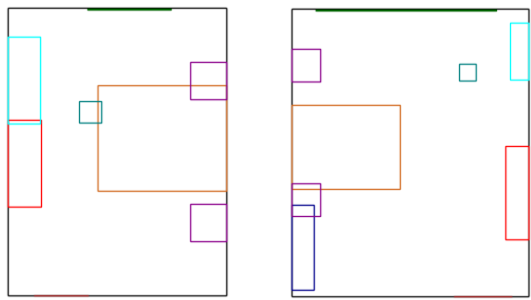

c. 去掉图神经网络

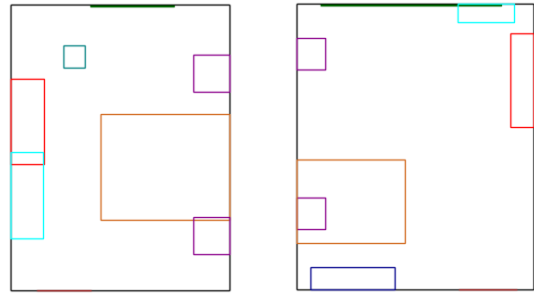

d. 去掉相对损失函数

图 4 消融实验的视觉结果 


\section{4 结 语}

本文通过图神经网络实现了将零散的家具摆 放成合理的室内场景布局。通过条件式变分自编 码器，本文算法具有生成多样式家具摆放方案的 效果，然而在复杂的室内场景中仍然具有较大的 局限性，对于训练集中不常见的厨房和卫生间等 房间类型也难以生成合理的布局结构. 在未来的 工作中, 可以通过进一步学习优化图网络的结构 来实现对复杂和不常见场景的家具摆放方案.

\section{参考文献(References):}

[1] Xu K, Stewart J, Fiume E. Constraint-based automatic placement for scene composition[C] //Proceedings of Graphics Interface. Calgary: Canadian Human-Computer Communications Society Press, 2002: 25-34

[2] Merrell P, Schkufza E, Li Z Y, et al. Interactive furniture layout using interior design guidelines[J]. ACM Transactions on Graphics, 2011, 30(4): Article No.87

[3] Yu L F, Yeung S K, Tang C K, et al. Make it home: automatic optimization of furniture arrangement[J]. ACM Transactions on Graphics, 2011, 30(4): Article No.86

[4] Fisher M, Ritchie D, Savva M, et al. Example-based synthesis of 3D object arrangements[J]. ACM Transactions on Graphics, 2012, 31(6): Article No.135

[5] Fu Q, Chen X W, Wang X T, et al. Adaptive synthesis of indoor scenes via activity-associated object relation graphs[J]. ACM Transactions on Graphics, 2017, 36(6): Article No.201

[6] Fisher M, Hanrahan P. Context-based search for 3D models[J]. ACM Transactions on Graphics, 2010, 29(6): Article No.182

[7] Xu K, Chen K, Fu H B, et al. Sketch2Scene: sketch-based co-retrieval and co-placement of 3D models[J]. ACM Transactions on Graphics, 2013, 32(4): Article No.123

[8] Ma R, Patil A G, Fisher M, et al. Language-driven synthesis of 3D scenes from scene databases[J]. ACM Transactions on Graphics, 2019, 37(6): Article No.212

[9] Chang A, Monroe W, Savva M, et al. Text to 3D scene generation with rich lexical grounding[C] //Proceedings of the 53rd Annual Meeting of the Association for Computational Linguistics and the 7th International Joint Conference on Natural Language Processing. Stroudsburg: Association for Computational Linguistics, 2015: 53-62

[10] Chen K, Lai Y K, Wu Y X, et al. Automatic semantic modeling of indoor scenes from low-quality RGB-D data using contextual information[J]. ACM Transactions on Graphics, 2014, 33(6): Article No.208

[11] Sadeghipour Kermani Z, Liao Z, Tan P, et al. Learning 3D scene synthesis from annotated RGB-D images[J]. Computer Graphics Forum, 2016, 35(5): 197-206

[12] Fisher M, Savva M, Li Y Y, et al. Activity-centric scene synthesis for functional 3D scene modeling[J]. ACM Transac- tions on Graphics, 2015, 34(6): Article No.179

[13] Song S R, Yu F, Zeng A, et al. Semantic scene completion from a single depth image[C] //Proceedings of the IEEE Computer Society Press Conference on Computer Vision and Pattern Recognition. Los Alamitos: IEEE Computer Society Press, 2017: 1746-1754

[14] Ritchie D, Wang K, Lin Y A. Fast and flexible indoor scene synthesis via deep convolutional generative models[C] // Proceedings of the IEEE Computer Society Press Conference on Computer Vision and Pattern Recognition. Los Alamitos: IEEE Computer Society Press, 2019: 6182-6190

[15] Wang K, Lin Y A, Weissmann B, et al. PlanIT: planning and instantiating indoor scenes with relation graph and spatial prior networks[J]. ACM Transactions on Graphics, 2019, 38(4): Article No.132

[16] Wang K, Savva M, Chang A X, et al. Deep convolutional priors for indoor scene synthesis[J]. ACM Transactions on Graphics, 2018, 37(4): Article No.70

[17] Li M Y, Patil A G, Xu K, et al. GRAINS: generative recursive autoencoders for indoor scenes[J]. ACM Transactions on Graphics, 2019, 38(2): Article No.12

[18] Zhang Z W, Yang Z P, Ma C Y, et al. Deep generative modeling for scene synthesis via hybrid representations[J]. ACM Transactions on Graphics, 2020, 39(2): Article No.17

[19] Zhou Y, While Z, Kalogerakis E. SceneGraphNet: neural message passing for 3D indoor scene augmentation[C] // Proceedings of the IEEE Computer Society Press International Conference on Computer Vision. Los Alamitos: IEEE Computer Society Press, 2019: 7384-7392

[20] Luo A, Zhang Z T, Wu J J, et al. End-to-end optimization of scene layout[C] //Proceedings of the IEEE Computer Society Press Conference on Computer Vision and Pattern Recognition. Los Alamitos: IEEE Computer Society Press, 2020: 3754-3763

[21] Krishna R, Zhu Y K, Groth O, et al. Visual Genome: connecting language and vision using crowdsourced dense image annotations[J]. International Journal of Computer Vision, 2017, 123(1): 32-73

[22] Li Y K, Ouyang W L, Zhou B L, et al. Scene graph generation from objects, phrases and region captions $[\mathrm{C}]$ //Proceedings of the IEEE Computer Society Press International Conference on Computer Vision. Los Alamitos: IEEE Computer Society Press, 2017: 1261-1270

[23] Johnson J, Gupta A, Li F F. Image generation from scene graphs[C]//Proceedings of the IEEE Computer Society Press Conference on Computer Vision and Pattern Recognition. Los Alamitos: IEEE Computer Society Press, 2018: 1219-1228

[24] Chen V S, Varma P, Krishna R, et al. Scene graph prediction with limited labels[C] //Proceedings of the IEEE Computer Society Press International Conference on Computer Vision. Los Alamitos: IEEE Computer Society Press, 2019: 2580-2590

[25] Krishna R, Chami I, Bernstein M, et al. Referring relationships[C] //Proceedings of the IEEE Computer Society Press Conference on Computer Vision and Pattern Recognition. Los Alamitos: IEEE Computer Society Press, 2018: 
6867-6876

[26] Xu D F, Zhu Y K, Choy C B, et al. Scene graph generation by iterative message passing[C] //Proceedings of the IEEE Computer Society Press Conference on Computer Vision and Pattern Recognition. Los Alamitos: IEEE Computer Society Press, 2017: 5410-5419

[27] Goodfellow I, Pouget-Abadie J, Mirza M, et al. Generative adversarial nets[C] //Proceedings of Advances in Neural Information Processing Systems. Cambridge: MIT Press, 2014: 2672-2680

[28] Kingma D P, Welling M. Auto-encoding variational Bayes[OL]. [2020-06-30]. https://arxiv.org/abs/1312.6114v10

[29] Sohn K, Yan X C, Lee H. Learning structured output repre- sentation using deep conditional generative models[C] // Proceedings of the 28th International Conference on Neural Information Processing Systems. Cambridge: MIT Press, 2015: 3483-3491

[30] Huang Yuan, Bai Cong, Li Hongkai, et al. Image captioning based on conditional generative adversarial nets[J]. Journal of Computer-Aided Design \& Computer Graphics, 2020, 32(6): 911-918(in Chinese)

(黄远, 白琮, 李宏凯, 等. 基于条件生成对抗网络的图像 描述生成方法 [J]. 计算机辅助设计与图形学学报, 2020, 32(6): 911-918)

[31] Kingma D P, Ba J. Adam: a method for stochastic optimization[OL]. [2020-06-30]. https://arxiv.org/abs/1412.6980 